\title{
Improvement of the Content Transmission in Broadcasting Systems: Potential Proposal to Rayleigh and Rician Multichannel MIMO
}

\section{Systems}

\author{
Reinaldo Padilha \\ Yuzo Iano \\ Ana Carolina Borges Monteiro \\ Hermes José Loschi
}




\title{
Improvement of the Content Transmission in Broadcasting Systems: Potential Proposal to Rayleigh and Rician Multichannel MIMO Systems
}

\author{
Reinaldo Padilha, Yuzo Iano, Ana Carolina B. Monteiro and Hermes José Loschi
}

\begin{abstract}
In the last years, the transmission of signals has been a widely approached theme, aiming the creation of methodologies that make channels increasingly efficient. Based on that, the present study implements a model based on discrete events applied to a broadcasting system entitled hybrid method, using the Simulink simulation environment of the MATLAB software. This study has the objective of improve the transmission of content, through a pre-coding process of bits applying discrete events in the signal before of the modulation process. This proposal brings a different approach, in which the signal transmission on the channel is realized in the discrete domain with the implementation of discrete entities in the process of bit generation. The results show better computational performance related to time and memory utilization related to the compression of the information, showing improvement 9.61 to $26,5 \%$, respectively.
\end{abstract}

Index Terms - discrete events, memory, simulation, bits.

\section{INTRODUCTION}

Computer simulations are excellent tool that supports the best knowledge of the behavior of a broadcasting system without building it, your results are accurate in general, compared to the analytic model. In the simulation environment, can be studied complex systems that would otherwise be difficult to investigate [1] [2] [9] [14] [15] [16]. As also can be used in engineering broadcasting to

R. Padilha is currently studying for a Ph.D.'s degree in Electrical Engineering, acting Laboratory of Visual Communications at the State University of Campinas (padilha@decom.fee.unicamp.br).

$\mathrm{Y}$. Iano is teacher and coordinator of the Laboratory of Visual Communications at the State University of Campinas (yuzo@decom.fee.unicamp.br). develop um proposal investigating the effect these changes without producing a physical prototype, providing the flexibility to implement different types of system architecture analyzing different layers, such as physical, transport, transmission and higher layers, improving and validating the system for different applications [1 - 9] [14 - 18].

The modeling Discrete Event is mainly used to represent the system being analyzed as a sequence of operations being performed on entities (transactions of state) of certain types such as data packets, bits, etc. The entities are discrete items of interest in a discrete event simulation. The meaning of an entity depends on what is being modeled and the type of system, and can have attributes that affect the way they are handled or may change as the entity flows through the process [1] [3] [5] [9 - 12].

Discrete events are the results of actions that occur in the system, an event that changes its state, being these actions intentional, spontaneously controlled or with the verification of a condition [3] [37 - 42].

This technique has been used to model concepts with a high-level of abstraction, as patients in a hospital in the healthcare system, clients in a queue in bank system, emails on a server and/or transmission of data packets in telecommunication's systems, etc. [2] [9 - 16].

Through of academic records, can be verified modeling of high-level of abstraction concepts such as people in business process systems, computers in computer networking systems, nodes in networks of data or telephone communication, vehicles in transport systems, service providers such as banks, transaction systems for databases, communication protocols in telecommunication's systems, people in call centers, vehicles in

A. C. B. Monteiro is currently studying for a Master's degree in Electrical Engineering, acting Laboratory of Visual Communications at the State University of Campinas (monteiro@decom.fee.unicamp.br).

H. J. Loschi is currently studying for a Ph.D.'s degree in Electrical Engineering, acting Laboratory of Visual Communications at the State University of Campinas (hermes@decom.fee.unicamp.br). 
intelligent transport systems, process control in control engineering, military equipment in defense systems, as many others [19 - 36]. In this way, are clear that the technique of modeling of discrete events is generally used to model concepts with a high-level of abstraction [42].

In this study, the authors developed a hybrid model for broadcasting and for radio broadcasting using an AWGN (Additive White Gaussian Noise) channel with advanced modulation format DQPSK (Differential Quadrature Phase Shift Keying) in simulation environment [42]. This study has the objective of to increase the transmission capacity of information content through of the channel.

Which a bit treatment with discrete events methodology was modeled in the step of bit generation, being the differential of this paper the use of discrete events applied in the physical layer of a transmission channel, being this a low-level of abstraction [42], reaching the second objective this paper.

The present paper is organized as follows: Section 2 discusses traditional simulation models, showing the modeling of transmission channel AWGN. Section 3 presents and describes the proposed framework of this paper, based on the hybrid model with discrete event methodology. Section 4 presents the results and, finally, in Section 5, the conclusions are presented as also the potential of the research.

\section{TRADITIONAL MODEL}

The communication channel is the medium that provides the physical connection between transmitters and receivers in a communication system, be it as a wire, or to a logical connection over a multiplexed medium such as a radio channel in telecommunications and computer networking. Carrying data typically uses two types of media: cable (twisted-pair wire, cable, and fiber-optic cable) and broadcast (microwave, satellite, radio, and infrared). For the analysis and development of proposals for improvement of communication systems, it is important to construct mathematical models that describe the main characteristics of these means and of the changes it makes to the signals transmitted [6] [7] [8] [17] [18].

An AWGN channel is a model practical of a communication system widely used due to its simplicity and mathematical treatment. It applies to a large set of physical channels, which introduces in the transmitted signals a noise modeled statistically as a white Gaussian additive process.

In the context of wireless communications, the main source of thermal noise is the addition of random signals arising from the vibration of atoms in the receiver electronics.

So, use the AWGN in these communication channels having a statistically random radio noise characterized by a wide frequency range with regard to a signal in the communications channel.

Systems operating largely in the AWGN conditions can be exemplified as space communications with highly directional antennas and some point-to-point microwave links.

The modulation formats QPSK (Quadrature Phase Shift Keying) and DPSK are widely used in satellite broadcasting, in various cellular wireless standards such as GSM, CDMA, LTE, 802.11 WLAN, 802.16 fixed and mobile WiMAX, Satellite as also CABLE TV applications. Being that the first is used in the streaming of SD satellite channels and some HD channels, however, the second has higher bit rates of HD video and a high satellite bandwidth, as also DQPSK can be significantly simpler to implement than QPSK [6] [7] [8] [17] [18].

Both DQPSK and QPSK are modulation formats for Digital Video and Radio Broadcasting, the DQPSK modulation is a particular form of QPSK modulation, in which instead of being sent a symbol corresponding to a pure phase parameter, this symbol represents a phase change [1] [4] [8] [17] [18].

In QPSK, the information is transmitted by the absolute phase of each symbol. Already on DQPSK, each set of bits represented by a symbol cause a determined phase variation in the carrier signal, in it, the bits for the data symbols are determined based on the phase change of the previous symbol [4] [8].

In this way, there are four possible states $0, \pi$, $+\pi / 4,-\pi / 4$. Thus, each symbol represents two bits of information. The division of the binary pattern is equal to QPSK, except that a bit string is shifted in phase about of $\pi / 4$ or $\pi / 2$ depending on the system [4] [8].

This means there is a total of 8 ideal state positions (compared to the 4 states for QPSK). The ideal state positions for symbols alternate between the four states of 45 degrees normally used by QPSK and four states on the axis. Due to this alternation, the ideal trajectory between symbols never crosses zero [1] [4] [8]. 
This session is presented a broadcasting system modeled with an AWGN channel with DQPSK modulation. For this, the authors used the Simulink simulation environment of the MATLAB ${ }^{\circledR}$ software in its version 8.3 of 64 bits (2014a).

In the model, Figure 01, the signals corresponding to the bits 0 and 1 are generated, and then modulated in DQPSK, following for an AWGN channel according to the parameters specified as sample time of 1 second, power input signal of 1 watt, initial seed in the generator of 37 and in the channel of $67, \mathrm{~Eb} / \mathrm{No}$ of 0 to $14 \mathrm{~dB}$. Then the signal is demodulated in order to perform the bit error rate (BER) of the channel. The values obtained referring to the BER are sent to the MATLAB workspace, for further processing and generating of the signal BER graph [42].

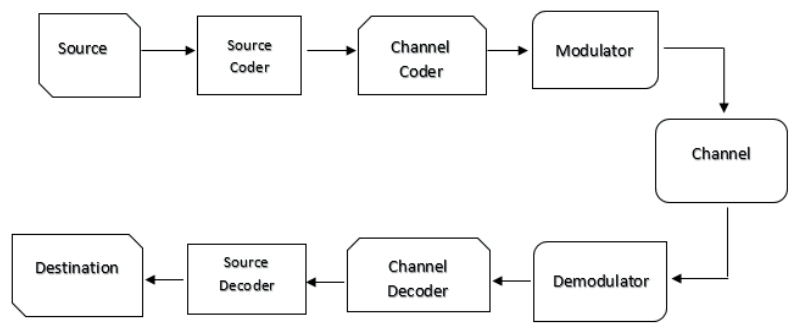

Figure 01 - Traditional Model

\section{PROPOSAL}

\section{A. Proposal}

The modeling of the pre-coding process according to proposal implemented with discrete events is similar with that shown in the previous section. Differentiating that in this model, was added the discrete events process of pre-coding, consisting of the treatment performed on the signal corresponding bit 0 . The bit 0 was converted into discrete entities, and forwarded for an FIFO queue with infinite capacity, without limit of capacity and retention in the block. The storing entities in the First-In-First-Out sequence, orders the bits following really your order of arrival, and thus, driving to a server. This server has the configuration of service time equal to the simulation time [42].

The differential of this paper is in the use of discrete events applied in such low-level of abstraction, being the bit generation. After the signal passes through the server, is converted back to its original format respecting the original format and data type specified and maintaining the sampling period respectively. Thus, the signal is modulated in DQPSK and inserted into the AWGN channel, and then demodulated for the purposes of calculating the BER of the signal. The relative values of this BER are also sent to the MATLAB workspace, for further processing and generating of the signal BER graph [42].

The model presented in Figure 02, incorporates the traditional modeling with a proposal presented, as well as highlights the part modeled according to the approach of discrete events, in blue, as previously described [42].

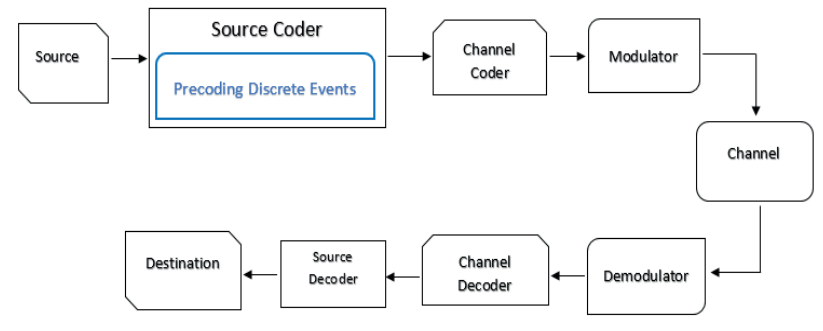

Figure 02 - Hybrid Model

In Figure 03, was used 10000 seconds of simulation time, being placed the flows of transmission of the DQPSK signal in relation to the hybrid model (below) and traditional model (top) for better viewing and comparison, noting that both methodologies generated the same result.
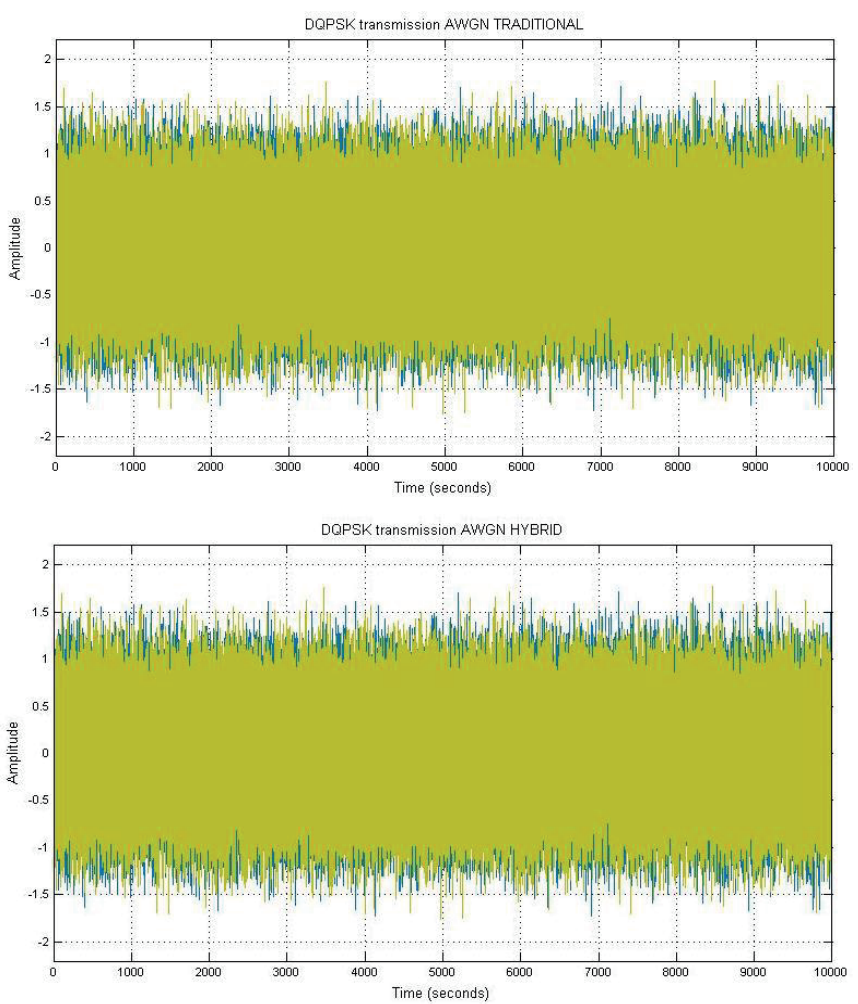

Figure 03 -Transmission Flow DQPSK 
The Constellation Diagram was used to view the constellation of the modulated digital signal also being useful for comparing the performance of one system with another [42].

In Figure 04 is shown the results for visualization of the constellations in 5,10 and 15 $\mathrm{dB}$, according to the hybrid model (below) and traditional model (top).
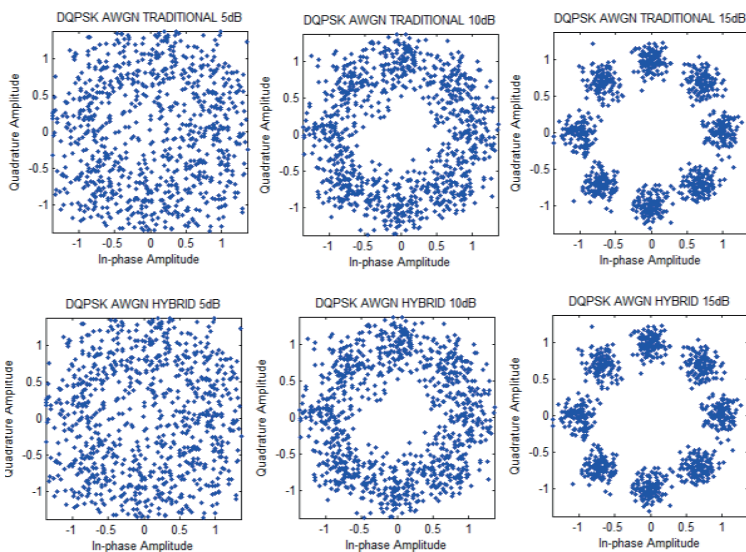

Figure 04 - Simulated DQPSK Constellations

\section{B. Proposal with Rayleigh and Rician}

The authors also applied to the proposal presented in a radio broadcasting system, where the mobile wireless channel is susceptible to several impediments including multipath, fading, shadowing, noise among other interferences. In such a way that these deficiencies can cause an enormous degradation in the performance of the system [6] [7] [8].

Rayleigh fading is a useful model of real-world phenomena in wireless communications. Characterized as a statistical model for the propagation effect in an environment with radio signal, is considered a reasonable and ideal model for heavily built urban environments with the propagation of signals in a means where there is no dominant propagation along a line of sight between the transmitter and the receiver [7] [8]

Rician fading is also a useful model of realworld phenomena in wireless communications. Characterized as a stochastic model for the propagation anomaly of the radio signal, caused by the partial cancellation of a radio signal by itself. When the signal reaches the receiver displaying multipath interference, at least one of the paths is changing (lengthening or shortening), and occurs when one of the paths, typically a lineof-sight signal, is much stronger than the others [6] [7] [8].

Generally, wireless networks differ mainly in their physical layer, wherein the transmission of data electromagnetic waves are used that propagate through space. Thus, for this type of transmission an efficient carrier frequency data modulation is required [6] [7] [8].

Within this context was modeled following the pattern of Figure 01, the signals corresponding to bits 0 and 1 are generated and then modulated in DQPSK. Posteriorly it is passed through a multipath Rayleigh fading channel and other with multipath Rayleigh fading, both containing Jakes model with Doppler shift defined at $0.01 \mathrm{~Hz}$, as also inserted a block incorporated which has a math function $1 / \mathrm{u}$ [42].

Such a function is required to track the timevariability channel where the receiver implementation ordinarily incorporates an automatic gain control (AGC). After is followed to an AWGN channel, according to the parameters specified as sample time of 1 second, power input signal of 1 watt, initial seed in the generator of 37 and in the channel of $67, \mathrm{~Eb} / \mathrm{No}$ of 0 to $14 \mathrm{~dB}$. Then the signal is demodulated in order to perform the bit error rate (BER) of the channel. The values obtained referring the BER are sent to the MATLAB workspace, for further processing and generating of the signal BER graph [42].

So, applied the same approach in relation to the pre-coding of the bits, noting the same result regarding the transmission flow and the constellations of the systems, validating the proposal [42], as shown in the Figures 05 to 08.
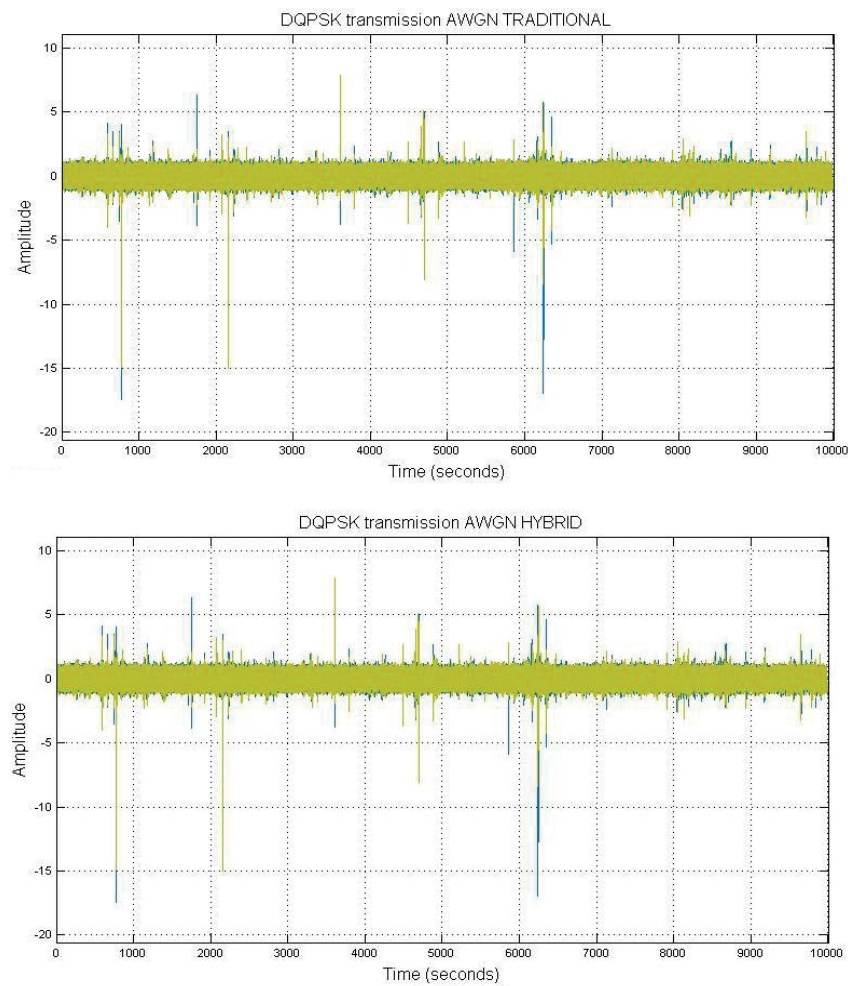

Figure 05 -Transmission Flow Rayleigh DQPSK 

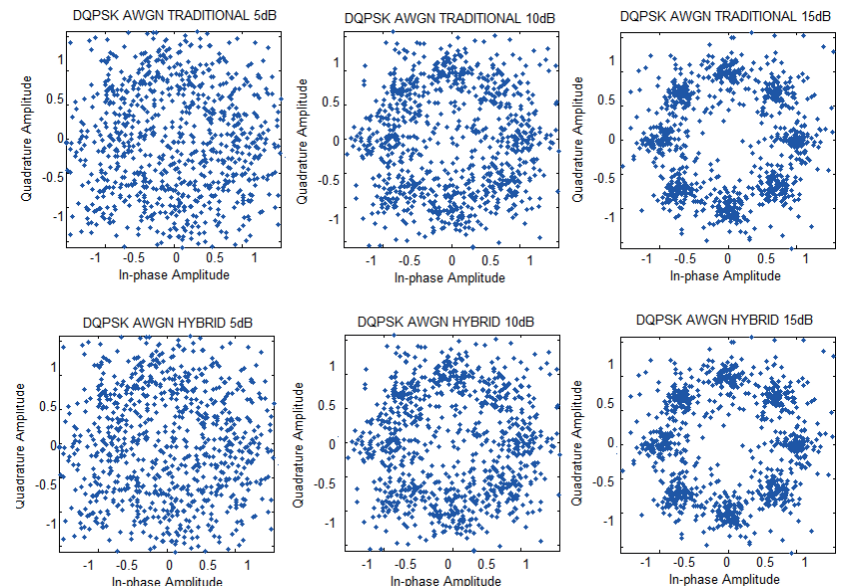

Figure 06 - Simulated DQPSK Constellations Rayleigh
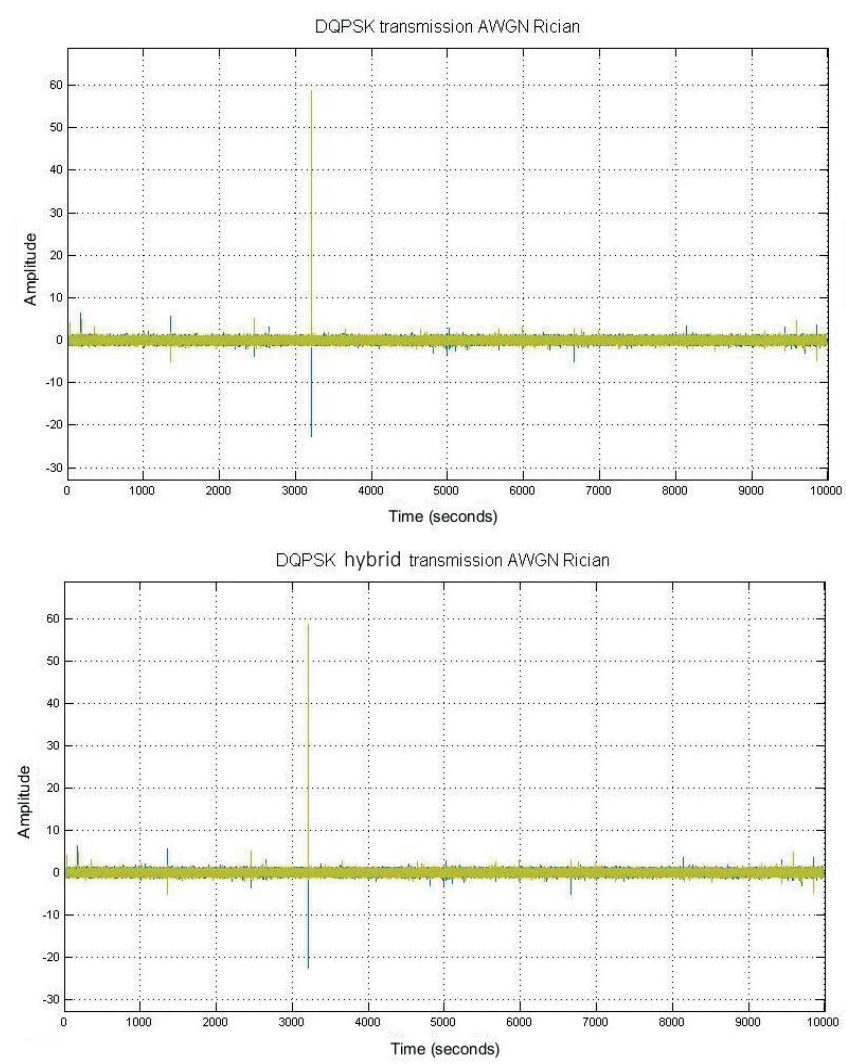

Figure 07 -Transmission Flow Rician DQPSK
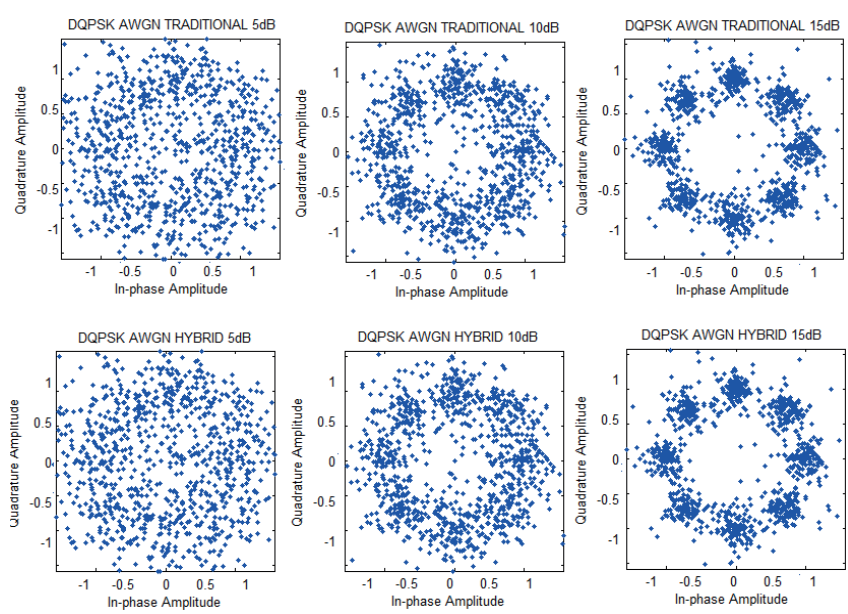

Figure 08 - Simulated DQPSK Constellations Rician

\section{RESULTS}

In this section, the results will be presented in relation to the evaluations of the time and memory consumption of the models according to the techniques studied and presented in the previous session. Also, will be presented a comparison among them, simulated on physical machines with different hardware configuration, consisting of an Intel Core i5 processor and 8GB RAM, and another with an Intel Core i3 processor and 4GB RAM [42].

The authors used the commands "tic", "toc", and "sim", via command line at the MATLAB's prompt, this measuring how much time the model takes to do the simulation (in seconds), that is, the simulation runtime [42].

The "sldiagnostics" function displays information about the modeling system in Simulink. This function is responsible to calculate the time spent on the simulated model, being this, the responsible to the accounting of the time spent in each phase of the simulation of the model (in seconds), that is, the simulation runtime [42].

This function also calculates the sum of all the memory consumption processes used in the model in simulation, by the ProcessMemUsage parameter. This parameter counts the amount of memory utilized in each phase of the model, during the entire simulation, displaying the total amount in MB [42].

In the same way, the authors analyzed the first simulation of both models in each command, and more the "cputime" command, which returns the total CPU time (in seconds). This command refers to the computation time, used by the application in use in MATLAB from the moment it was started. It's important because it is the first simulation that the variables are allocated and the memory reserved for the execution of the model [42], according presented in the Figures 09 to 11. 


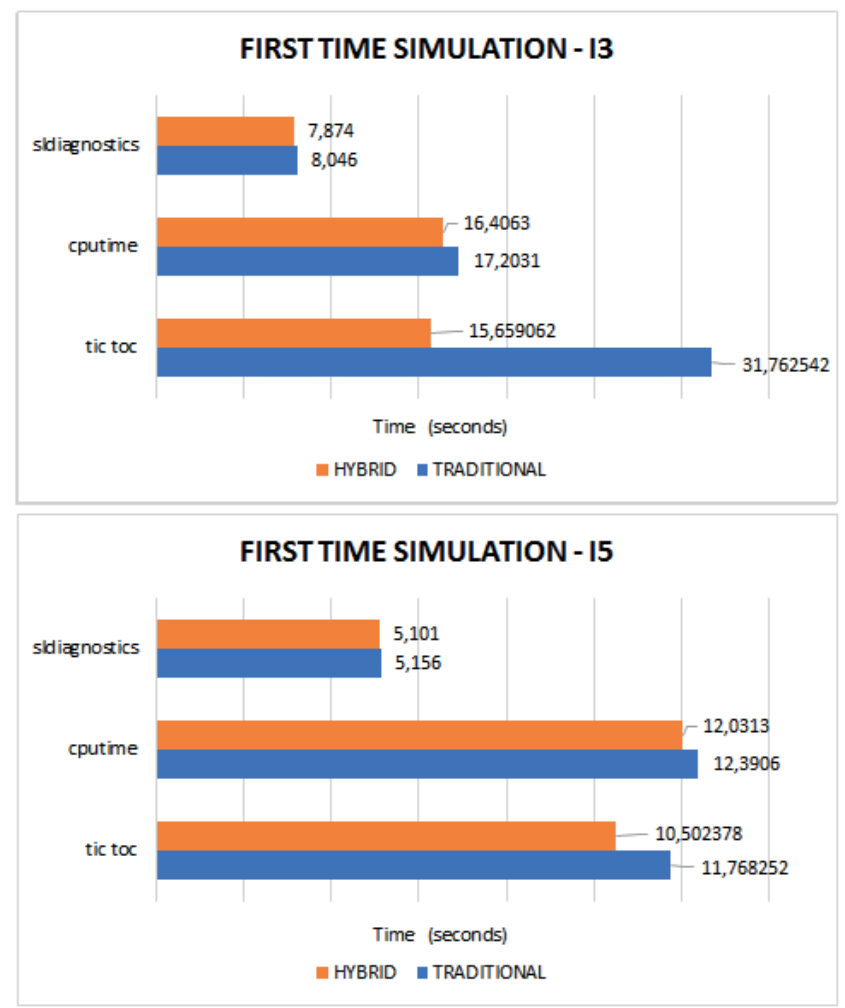

Figure 09 - First-Time Simulation

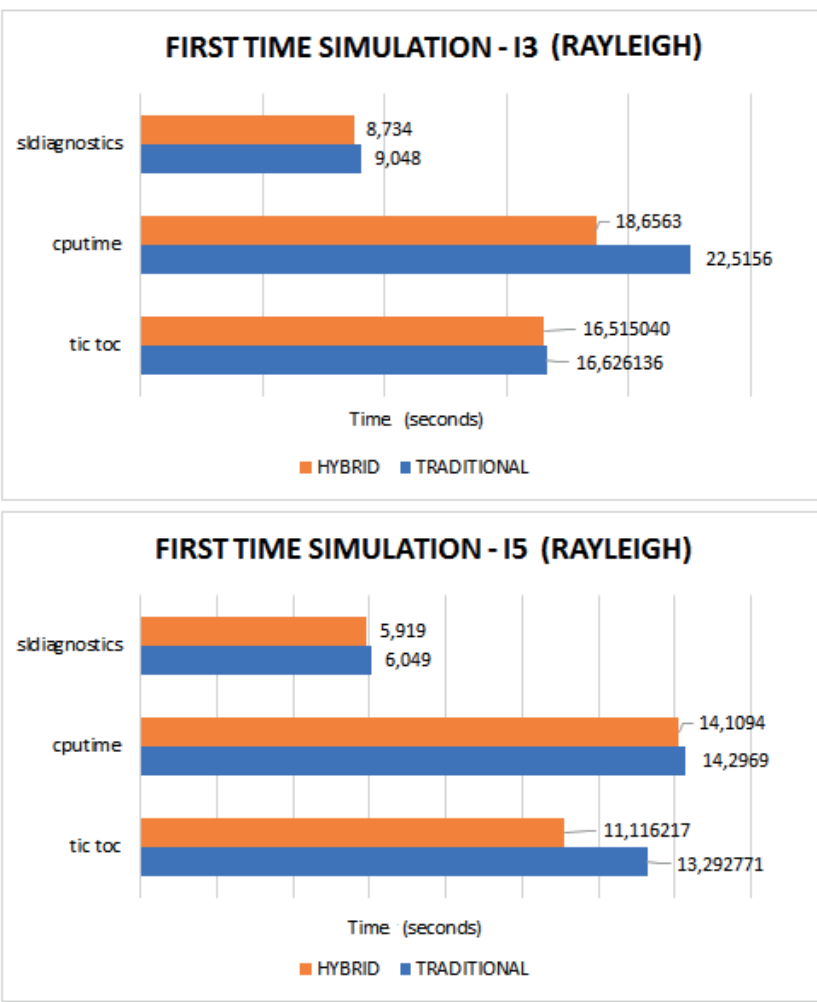

Figure 10 - Rayleigh First-Time Simulation

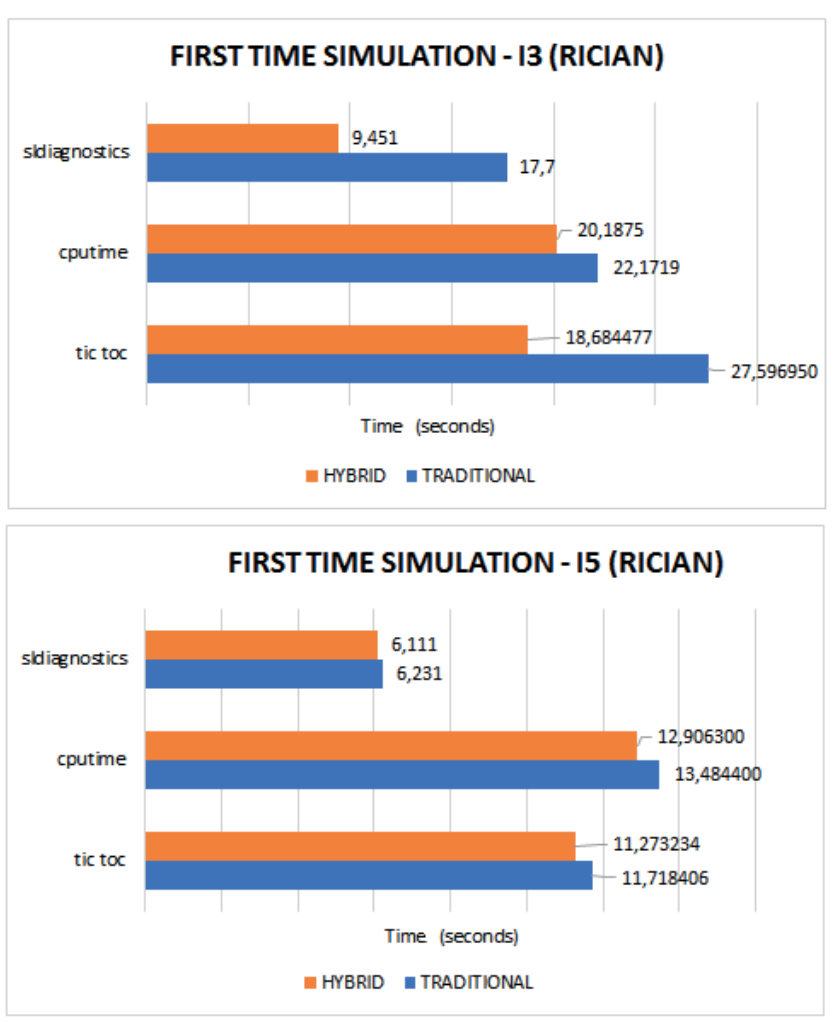

Figure 11 - Rician First-Time Simulation

Similarly, also was observed the first simulation of the models regarding their memory consumption [42], having a better performance as shown in TABLE I and related with the Figures 12 to 14 .

TABLE I. COMPUTATIONAL IMPROVEMENT

\begin{tabular}{|c|c|c|}
\hline \multicolumn{3}{|c|}{ Memory Consumption / Rate Compression } \\
\hline Machines & $\mathbf{i 3}$ & $\mathbf{i 5}$ \\
\hline Broadcasting DQPSK & $24,20 \%$ & $9.61 \%$ \\
\hline $\begin{array}{c}\text { Broadcasting DQPSK } \\
\text { Rayleigh }\end{array}$ & $26,59 \%$ & $9.51 \%$ \\
\hline $\begin{array}{c}\text { Broadcasting DQPSK } \\
\text { Rician }\end{array}$ & $22,85 \%$ & $11,65 \%$ \\
\hline
\end{tabular}

Can be understood that if in a transmission channel containing the proposal and in another the traditional methodology, they passed the same information content (quantity of bits), without any loss (signal and constellation) and with the same quality (BER). The result related to the memory consumption of the proposal is relative to the compression of the information [42], as shown in TABLE I and related with the Figures 12 to 14 . 




Figure 12 - First Memory Consumption Simulation

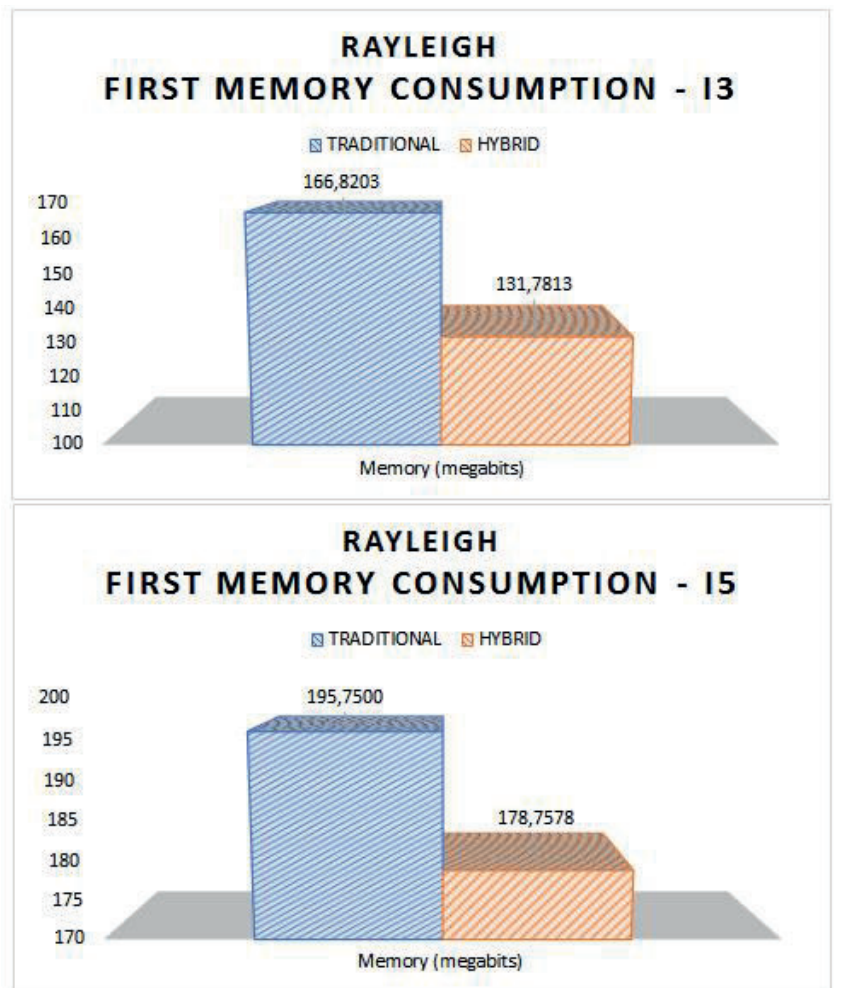

Figure 13 - Rayleigh First Memory Consumption Simulation

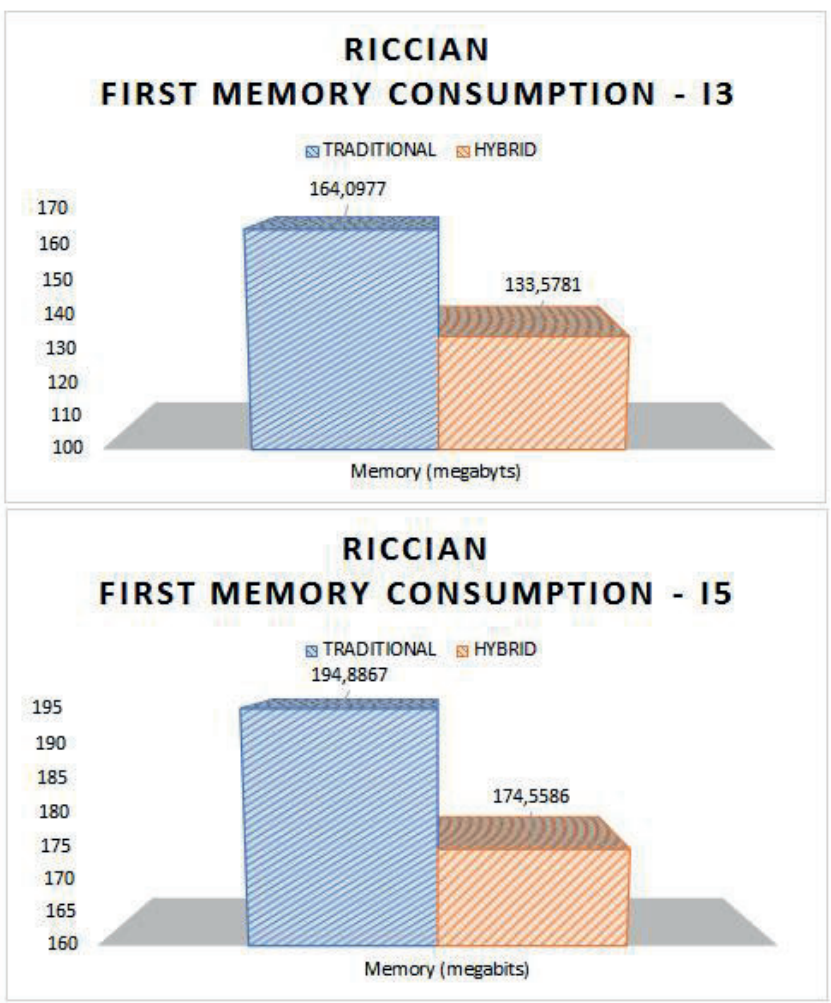

Figure 14 - Rician First Memory Consumption Simulation

Also, is noted that the proposal reduced practically all the memory consumption resulting from Rayleigh and Rician multipath fading in models simulated with such distributions and in both machines. Equalizing its resource consumption to a channel without the Rayleigh and Rician techniques, preserving all the benefits and characteristics of propagation across multipaths [42], as shown in the Figures 15 and 16.

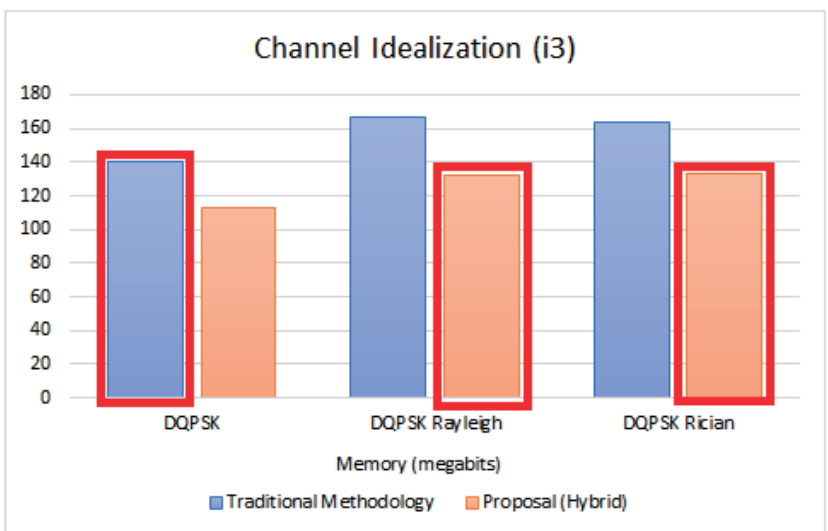

Figure 15 - Channel Idealization i3 


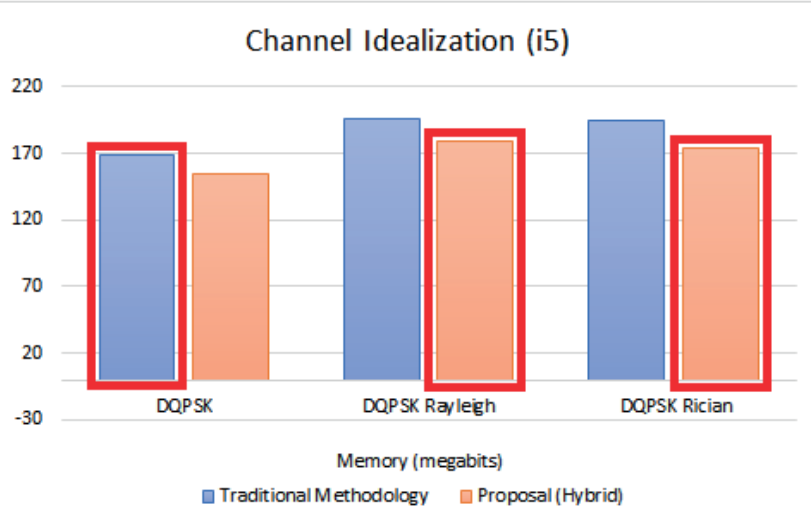

Figure 16 - Channel Idealization i5

To analyze the relationship between the simulation methodology and the impact on the physical layer of the channel, scripts were made in the MATLAB workspace for processing of the graph relative to BER. This allows analyzing the performance of bit error rate (BER) in communication's systems [42].

In the Figures 17 to 19 , is displayed the performance of the models according to simulation methodologies under study, along with a transmission with noise ranging from 0 to $60 \mathrm{~dB}$.

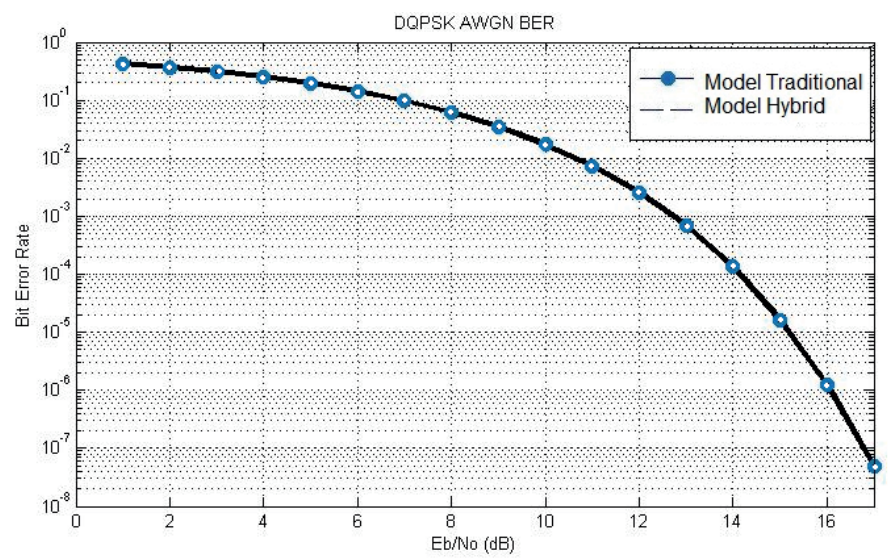

Figure 17 - Performance BER

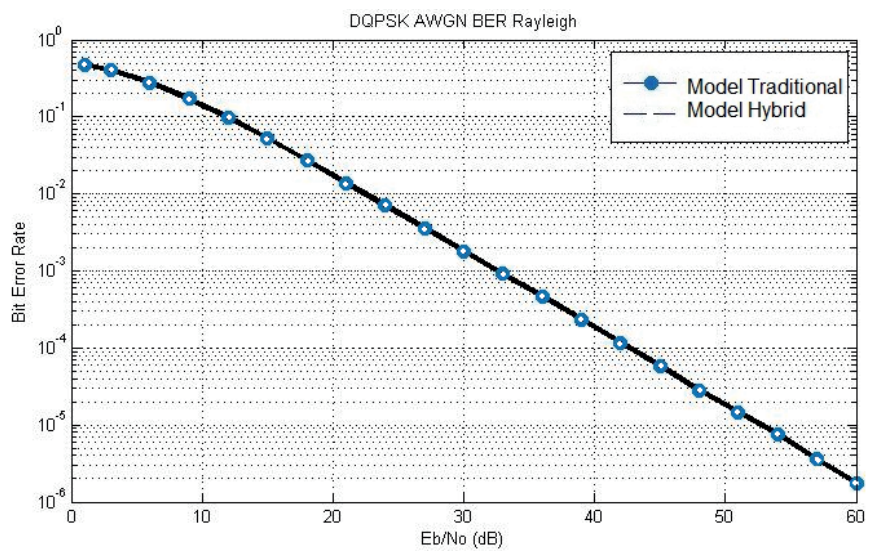

Figure 18 - Performance Rayleigh BER



Figure 19 - Performance Rician BER

\section{CONCLUSIONS}

The use of discrete events applied in a lowlevel of abstraction such as the bit, in generation phase in a broadcasting system, was the differential of this research since it generally does not apply discrete events in this way.

Evaluating the results, show that the simulation models of broadcasting systems taken a different approach from what is normally done. This proposal applied a concept of a methodology, naturally applied at higher levels, in a lower abstraction level, in bits in the transmission of a channel, through discrete entities

In all scenarios and prospects analyzed the model of the broadcasting system, where was applied the proposal with discrete events show consistent results in the simulated virtual environment. The results obtained on different hardware configurations, evaluated memory consumption and simulation time, and show better performance compared with the model that had only with the traditional methodology.

One extension of the results of this research, being her the compression of the information, strongly affects similar methods performed in higher layers, like MPEG-4 for example, as well as others, can improve them even more since this proposal acts on the bits.

The results related to channel idealization showed the potential of the proposal on Rayleigh and Rician multi-channel MIMO systems, where it is considered within the context of NOMA (Non-Orthogonal Multiple Access), future cellular radio access, $5 \mathrm{G}$.

The purpose of this research together with the proposal presented in this paper is to contribute to the study area and in growing development, the broadcasting. 


\section{REFERENCES}

[1] Digital Modulation in Communications Systems - An Introduction, Agilent Technologies

[2] Forrester, J.W., 1968. 'Industrial Dynamics - After the First Decade'. Management Science, 14(7), pp. 398415.

[3] Helal, M. 2008. “A Hybrid System Dynamics-Discrete Event Simulation Approach to Simulating the Manufacturing Enterprise." Ph.D. Thesis, Department of Industrial Engineering and Management Systems, College of Engineering and Computer Science, University of Central Florida.

[4] L.W. Couch II, Digital and Analog Communication Systems, 8th Edition, Prentice Hall, 2013.

[5] Padilha, R. and Martins, B. I. and Moschim, E., 2016. "Discrete Event Simulation and Dynamical Systems: A study of art". BTSym'16, Campinas, SP - Brasil, December 2016

[6] Roger L. Freeman, Fundamentals of Telecommunications, John Wiley \& Sons, 1999.

[7] Roger L. Freeman, Telecommunication System Engineering, 4th Edition, John Wiley \& Sons, 2004.

[8] John G. Proakis (2008). Digital Communications, 5th edition, McGraw-Hill.

[9] Pereira, F. T., Takano, A. M., Leal, F.and Pinho, F. A., 2013. "Aplicação Da Simulação A Eventos Discretos Em Um Ambiente Hospitalar Visando A Melhoria No Processo De Atendimento". XLVSBPO, Natal, RN Brasil.

[10] Pissinelli, J. G., Risso, L. A., Picanco, S. R. A., Ignacio, A. S. P. and Silva, L. A. 2015. "Modelo De Simulação De Eventos Discretos Para Análise De Fluxo De Veículos". ENEGEP, Fortaleza, CE - Brasil.

[11] Rangel, J.J.A., Costa, J.V.S., Laurindo, Q.M.G., Peixoto, T.A. and Matias, I.O. "Análise do fluxo de operações em um servidor de e-mail através de simulação a eventos discretos com o software livre Ururau". Produto \& Produção, vol. 17, n. 1, p. 1-12, mar. 2016

[12] Gomes, E.N., Fernandes, M.S.R., Campos, C.A.V., Viana, A.C. "Um Mecanismo de Remoção de Mensagens Obsoletas para as Redes Tolerantes a Atrasos e Interrupções". CSBC, 2012.

[13] Godoy, E.P., Lopes, W.C., Sousa, R.V. and Porto, A.J.V. "Modelagem E Simulação De Redes De Comunicação Baseadas No Protocolo Can - Controller Area Network". Revista SBA: Controle \& Automação, Vol.21 no.4, 2010.

[14] Sharda, B and Bury, J. S. 2008. "A Discrete Event Simulation Model For Reliability Modeling Of A Chemical Plant". Winter Simulation Conference.

[15] Hu, W., Sarjoughian, H.S. 2005. "Discrete-event simulation of network systems using distributed object computing". SPECTS'05.

[16] Sasaki, N. K. and Moschim, E. 2007. "Simulação de Sistemas de Comunicação Óptica Baseada em Simulação a Eventos Discretos". Universidade Estadual de Campinas. Campinas, SP - Brasil. July 2007.

[17] TOZER, E. P., Broadcast Engineer's Reference Book, 1th Edition, FOCAL PRESS, 2012

[18] Whitaker C. J., Standard Handbook of Broadcast Engineering, 1th Edition, McGraw-Hill, 2005

[19]Zhao, B., Lin, F., Wang, C., Zhang, X., Polis, P. M, and Wang, Y. L., Supervisory Control of Networked Timed Discrete Event Systems and Its Applications to Power Distribution Networks, IEEE Transactions on Control of Network Systems (Volume: 4, Issue: 2, June 2017), 2017

[20] Wang, S., Nakayama, K., Kobayashi, Y. and Maekawat, M., A Data Schema and Its Query for Modeling Dynamic Geographical World, Communications and Information Technology, 2005. ISCIT 2005. IEEE International Symposium on

[21] Deb, S., Chowdhury, N. F. A. and Claudio, D., Service Quality Improvement in an IT Center: A Simulation
Study, Proceedings of the 2014 Industrial and Systems Engineering Research Conference, 2014

[22] Xu, X. and Wang, Z.; Networked Modeling and Simulation Based on SimEvents. System Simulation and Scientific Computing, 2008. IEEE ICSC 2008 Asia Simulation Conference - 7th International Conference on

[23] Li, F.; Wang, L. and Liao, C.; CAN (Controller Area Network) Bus Communication System Based on Matlab/Simulink, IEEE 2008 4th International Conference on Wireless Communications, Networkingand Mobile Computing

[24] Zelenka, J.; Discrete event dynamic systems framework for analysis and modeling of real manufacturing system, 2010 IEEE 14th International Conference on Intelligent Engineering Systems

[25] Pomante, L.; Spinosi, A.; Mostafizur, M.; Mozumdar, R. and S. O. L. Lavagno. An Extended Framework for the Development of WSN Applications. IEEE Ultra Modern Telecommunications and Control Systems and Workshops (ICUMT), 2010 International Congress on

[26] Bastos, G. S.; Souza, L. E.; Ramos, F. T. and Ribeiro, C. H. C.; A Single-dependent Agent Approach for Stochastic Time-Dependent Truck Dispatching in Open-pit Mining, 2011 14th International IEEE Conference on Intelligent Transportation Systems Washington, DC, USA. October 5-7, 2011

[27]Han, U. S. P. F.; Khalil, I. and Tari, K.; Preference Based Load Balancing As An Outpatient Appointment Scheduling Aid, 35th Annual International Conference of the IEEE EMBS Osaka, Japan, 3 - 7 July, 2013

[28] Ayoob, S. A. and Jabbar, A. I. A., Modeling a Multihop Ad-Hoc Network using Chain and CrossTopologies, The First IEEE International Conference of Electrical, Communication, Computer, Power and Control Engineering ICECCPCE'13. December 17-18, 2013

[29] Alsebae, A.; Leeson, M. and Green, R.; The Throughput Benefits of Network Coding for SW ARQ Communication. IEEE 2013 27th International Conference on Advanced Information Networking and Applications Workshops.

[30]Alsebae, A.; Leeson, M. and Green, R.; The Throughput Benefits of Network Coding for SR ARQ Communication. IEEE 2013 5th Computer Science and Electronic Engineering Conference (CEEC)

[31] Alsebae, A. A.; Leeson, M. S. and Green, R. J.; SimEvents-based Modeling and Simulation Study of Stop-and-Wait Protocol. IEEE 2013 5th International Conference on Modelling, Identification and Control (ICMIC)

[32] Alsebae, A.; Leeson, M. and Green, R.; Performance of a Network Coding Queuing Model with Deterministic Service. IEEE 2014 9th International Symposium on Communication Systems, Networks \& Digital Sign (CSNDSP)

[33] Mansouri, D.; Mokddad, L.; Ben-othman, J. and Ioualalen, M.; Preventing Denial of Service Attacks in Wireless Sensor Networks. 2015 IEEE International Conference on Communications (ICC)

[34] Magsino, E. R. and Ho, I. W. H.; An Intelligent Highway Tollgate Queue Selector for Improving Server Utilization and Vehicle Waiting Time. 2016 IEEE Region 10 Symposium (TENSYMP), Bali, Indonesia

[35] Shukla, O. J.; Soni, G.; Sujil, A. and Kumar, R.; Discrete Event System Framework for Analysis and Modeling of Job shop Scheduling System. IEE 2016 Intl. IEEE Conference on Advances in Computing, Communications and Informatics (ICACCI), Sept. 2124, 2016, Jaipur, India

[36] Hill, R. R., McIntyre, A. G., Applications of Discrete Event Simulation Modeling to Military Problems, 2001, Winter Simulation Conference

[37]K. Chahal and T. Eldabi, "A multi-perspective comparison between system dynamics and discrete event simulation," Journal of Business Information Systems, pp. 4-17, 2010 
[38] Brito, T. B., E. F. C. Trevisan, and R. C. Botter, 2011. A Conceptual Comparison Between Discrete and Continuous Simulation to Motivate the Hybrid Simulation Technology. In Proceedings of the 2011 Winter Simulation Conference, Edited by S. Jain, R.R. Creasey, J. Himmelspach, K.P. White, and M. Fu, 3915-3927

[39] Crespo-Márquez, A., R. R. Usano and R. D. Aznar, 1993, "Continuous and Discrete Simulation in a Production Planning System. A Comparative Study", Proceedings of International System Dynamics Conference, Cancun, Mexico, p. 58, The System DynamSociety

[40] Law, A., and W. Kelton. 2000. Simulation Modeling And Analysis. McGrawHill, USA

[41] Venkateswaran, J., Y. -J. SON*, 2005. 'Hybrid system dynamic - discrete event simulation-based architecture for hierarchical production planning'. International Journal of Production Research, Vol. 43, No. 20, 15 October 2005

[42] PADILHA, R. Proposta de Um Método Complementar de Compressão de Dados Por Meio da Metodologia de Eventos Discretos Aplicada Em Um Baixo Nível de Abstração. Dissertacão (Mestrado em Engenharia Elétrica) - Faculdade de Engenharia Elétrica e de Computação, Universidade Estadual de Campinas. Campinas, SP - Brasil. 2018

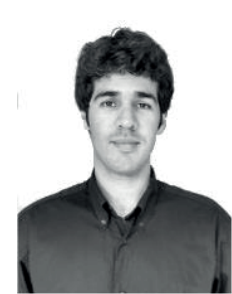

Reinaldo Padilha. Graduated in Computer Engineering (University Regional Center of Espírito Santo de Pinhal - 2014). Currently is a Ph.D. Candidate by Department of Communications (DECOM), Faculty of Electrical and Computer Engineering (FEEC) at State University of Campinas (UNICAMP), and a researcher at the Laboratory of Visual Communications (LCV). He also is currently Proceedings Chair of the Brazilian Symposium on Technology (BTSym). Has interest and affinity in the area of technological and scientific research as well as knowledge in programming and development in $\mathrm{C} / \mathrm{C}++$, Java and .NET languages. The main topics of interest are Simulation, Operating Systems, Software Engineering, Wireless and Network, Internet of Things, Broadcasting and Telecommunications Systems.

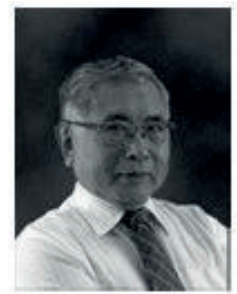

Prof ${ }^{0}$ Yuzo Iano. BS (1972), Master's degree (1974) and a Ph.D. degree (1986) in Electrical Engineering from the State University of Campinas, Brazil. Since then he has been working in the technological production field, with 1 patent granted, 8 patent applications filed and 36 projects completed with research and development agencies. Successfully supervised 29 doctoral theses, 49 master's dissertations, 74 undergraduate and 48 scientific initiation works. He has participated in 100 master's examination boards, 50 doctoral degrees, author of 2 books and more than 250 published articles. He is currently Professor at the State University of Campinas, Brazil, Editor-in-Chief of the SET International Journal of Broadcast Engineering and General Chair of the Brazilian Symposium on Technology (BTSym). He has experience in Electrical Engineering, with knowledge in Telecommunications, Electronics and Information Technology, mainly in the field of audio-visual communications and data.

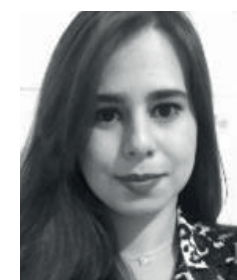

Ana Carolina Borges Monteiro. Graduated in Biomedicine from Centro Universitário Amparense - UNIFIA (2015). Currently is an MSc degree candidate by Department of Communications (DECOM), Faculty of Electrical and Computer Engineering (FEEC) at State University of Campinas (UNICAMP), and a researcher at the Laboratory of Visual Communications (LCV). She also is currently Registration Chair of the Brazilian Symposium on Technology (BTSym). Has expertise in the areas of Clinical Analysis and digital image processing through Matlab software. This knowledge was acquired through the realization of research projects and internship in municipal hospital, as also experience in the revision of scientific works by acting as a reviewer in congresses.

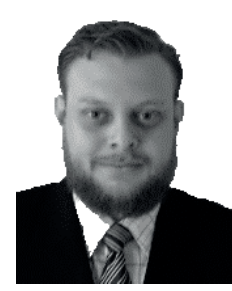

Hermes José Loschi. Graduated in Control and Automation Engineering, M.Sc in Electrical Engineering. Currently a Ph.D. Candidate by Department of Communications (DECOM), Faculty of Electrical and Computer Engineering (FEEC) at State University of Campinas (UNICAMP). He also is currently Technical Program and Finance Chair of the Brazilian Symposium on Technology (BTSym). The main topics of interest are Wireless Sensor Network, Internet of Things, Smart Grid, Broadcasting, Biomass, Photovoltaic Systems Applications, Solar Energy, Photovoltaic Solar Generation Prediction Systems, Solar Tracking, Open Access, and STEM. Reviewer for the following publishers: SCIENCEDOMAIN International, Elsevier, International Knowledge Press, Scientific Research Publishing, David Publishing Company, Canadian Center of Science and Education, PIBIC-PRP- UNICAMP. Student member of IEEE and ISDS. 